\title{
NONLOCAL SYMMETRIES, CONSERVATION LAWS, AND RECURSION OPERATORS OF THE VERONESE WEB EQUATION
}

\author{
I.S. KRASIL'SHCHIK, O.I. MOROZOV, AND P. VOJČÁK
}

\begin{abstract}
We study the Veronese web equation $u_{y} u_{t x}+\lambda u_{x} u_{t y}-(\lambda+1) u_{t} u_{x y}=0$ and using its isospectral Lax pair construct two infinite series of nonlocal conservation laws. In the infinite differential coverings associated to these series, we describe the Lie algebras of the corresponding nonlocal symmetries. Finally, we construct a recursion operator and explore its action on nonlocal shadows. The operator provides a new shadow which serves as a master-symmetry.
\end{abstract}

\section{Contents}

Introduction

1. The Veronese web equation and its coverings

1.1. The positive covering

1.2. The negative covering

2. Lie algebras of nonlocal symmetries

2.1. The algebra $\operatorname{sym}_{\tau^{+}}(\mathcal{E})$

2.2. The algebra $\operatorname{sym}_{\tau^{-}}(\varepsilon)$

3. Recursion operators and a master-symmetry

3.1. Action of recursion operators

3.2. Master-symmetry

Acknowledgments

References

Date: May 29, 2019.

2010 Mathematics Subject Classification. 35B06.

Key words and phrases. Partial differential equations, Veronese web equation, differential coverings, Lax pairs, nonlocal symmetries, recursion operators, master symmetries.

The work of IK was partially supported by the RFBR Grant 18-29-10013 and IUM-Simons Foundation.

The work of OM was partially supported by the Faculty of Applied Mathematics of AGH UST statutory tasks within subsidy of Ministry of Science and Higher Education.

The third author (PV) was supported by the Ministry of Education, Youth and Sports of the Czech Republic under the project CZ.02.2.69/0.0/0.0/16_027/0008521. 


\section{INTRODUCTION}

This work finalizes our research of Lax-integrable (i.e., admitting Lax pairs with non-vanishing spectral parameter) linearly degenerate (in the sense of [11]) 3D equations, see [1, 2, 3, 4, 12, 13. We deal here with the Veronese web equation (VWE)

$$
u_{y} u_{t x}+\lambda u_{x} u_{t y}-(\lambda+1) u_{t} u_{x y}=0,
$$

which is a generic case of the so-called ABC-equation, $A+B+C=0$, introduced in 26] (see also [10, [18]). Here $\lambda \neq 0$ is a real parameter. This equation determines three-dimensional Veronese webs that appear in the study of three-dimensional bi-Hamiltonian systems, see [8] and references therein. In [10] a one-to-one correspondence between three-dimensional Veronese webs and Lorentzian EinsteinWeyl structures of hyper-CR type was found. The latter are parametrized by the solutions of the hyper-CR equation

$$
u_{y y}=u_{t x}+u_{y} u_{x x}-u_{x} u_{x y},
$$

which is a symmetry reduction of Plebański's second heavenly equation, see [9]. In [23] it was shown that equations (11) and (2) are related by a Bäcklund transformation. This transformation produces, inter alia, non-local conservation laws for equation (2) from local conservation laws of equation (1), see [19].

Equation (10) admits a Lax pair with a non-vanishing spectral parameter [26], see also [7, and we use the scheme applied before in similar situations: we expand this Lax pair in formal series with respect to the spectral parameter and "cut" the result into two infinite-dimensional coverings called the positive and negative ones $\tau^{+}$and $\tau^{-}$, resp., see Section 1. Then, in Section 2, we give a full description of the Lie algebras formed by nonlocal symmetries in these coverings. The arising algebras are infinite-dimensional and possess quite an interesting structures, to our opinion. In Section 3. we construct two mutually inverse recursion operators and study their action on shadows of nonlocal symmetries. An interesting feature of the VWE which distinguishes it from other linearly degenerate equations is that the recursion operators generate a new symmetry in the Whitney product of $\tau^{+}$and $\tau^{-}$. This is a master-symmetry.

In the subsequent exposition, we use definitions and constructions from the geometric theory of PDEs [6] and its nonlocal version [16]. A concise exposition of the necessary material can be found in the preliminary parts of [12] or [4]. Everywhere below we omit the proofs that are accomplished by straightforward computations.

\section{The Veronese Web EQUation And its COVERINGS}

Rewrite Equation (11) in the form

$$
u_{t y}=\frac{(\lambda+1) u_{t} u_{x y}-u_{y} u_{t x}}{\lambda u_{x}}
$$

and choose the functions

$$
x, y, t, u_{x^{k}}, u_{x^{k} y^{l}}, u_{x^{k} t^{l}}, \quad k \geq 0, l>0,
$$

for internal coordinates on the infinite prolongation $\mathcal{E}$ of VWE, where

$$
u_{x^{i} y^{i} t^{k}}=\frac{\partial^{i+j+k} u}{\partial x^{i} \partial y^{j} \partial t^{k}} .
$$

Then the total derivatives on $\mathcal{E}$ acquire the form

$$
\begin{aligned}
D_{x} & =\frac{\partial}{\partial x}+\sum_{k} u_{x^{k+1}} \frac{\partial}{\partial u_{x^{k}}}+\sum_{k, l}\left(u_{x^{k+1} y^{l}} \frac{\partial}{\partial u_{x^{k} y^{l}}}+u_{x^{k+1} t^{l}} \frac{\partial}{\partial u_{x^{k} t^{l}}}\right), \\
D_{y} & =\frac{\partial}{\partial y}+\sum_{k} u_{x^{k} y} \frac{\partial}{\partial u_{x^{k}}}+\sum_{k, l}\left(u_{x^{k} y^{l+1}} \frac{\partial}{\partial u_{x^{k} y^{l}}}+D_{x}^{k} D_{t}^{l-1}(R) \frac{\partial}{\partial u_{x^{k} t^{l}}}\right), \\
D_{t} & =\frac{\partial}{\partial t}+\sum_{k} u_{x^{k} t} \frac{\partial}{\partial u_{x^{k}}}+\sum_{k, l}\left(D_{x}^{k} D_{y}^{l-1}(R) \frac{\partial}{\partial u_{x^{k} y^{l}}}+u_{x^{k} t^{l+1}} \frac{\partial}{\partial u_{x^{k} t^{l}}}\right),
\end{aligned}
$$

where $R$ is the right-hand side of (3). 
The Lax pair for the VWE is

$$
w_{t}=\frac{\mu(\lambda+1)}{\lambda(\mu+1)} \frac{u_{t}}{u_{x}} w_{x}, \quad w_{y}=\frac{\mu}{\lambda} \frac{u_{y}}{u_{x}} w_{x}
$$

where $\mu \in \mathbb{R}$ is the spectral parameter. Expanding $w=\sum_{i} \mu^{i} w_{i}$, one obtains

$$
w_{i, t}=\frac{(\lambda+1)}{\lambda} \frac{u_{t}}{u_{x}} w_{i-1, x}-w_{i-1, t}, \quad w_{i, y}=\frac{1}{\lambda} \frac{u_{y}}{u_{x}} w_{i-1, x}
$$

where $i \in \mathbb{Z}$. Setting $w_{i}=0$ for $i<0$, we obtain the positive covering; if we set $w_{i}=0$ for $i>0$ the negative covering arises.

1.1. The positive covering. The defining equations for the positive covering $\tau^{+}: \mathcal{E}^{+} \rightarrow \mathcal{E}$ are

$$
q_{i, t}=\frac{(\lambda+1)}{\lambda} \frac{u_{t}}{u_{x}} q_{i-1, x}-q_{i-1, t}, \quad q_{i, y}=\frac{u_{y} q_{i-1, x}}{\lambda u_{x}}
$$

where $i \geq 1$ and we formally set $q_{0}=x$. Nonlocal variables in the covering $\tau^{+}: \mathcal{E}^{+} \rightarrow \mathcal{E}$ are $q_{i, x}^{k}$, $i \geq 1, k \geq 0$ (in particular, $q_{i, x}^{0}=q_{i}$ ), and the total derivatives on $\mathcal{E}^{+}$are

$$
\tilde{D}_{x}=D_{x}+\sum_{i, k} q_{i, x}^{k+1} \frac{\partial}{\partial q_{i, x}^{k}}, \quad \tilde{D}_{y}=D_{y}+\sum_{i, k} \tilde{D}_{x}^{k}\left(q_{i, y}\right) \frac{\partial}{\partial q_{i, x}^{k}}, \quad \tilde{D}_{t}=D_{t}+\sum_{i, k} \tilde{D}_{x}^{k}\left(q_{i, t}\right) \frac{\partial}{\partial q_{i, x}^{k}},
$$

where $q_{i, y}$ and $q_{i, t}$ are given by Equations (7).

Consider the tower of coverings

$$
\mathcal{E}^{+} \longrightarrow \ldots \longrightarrow \varepsilon_{i+1}^{+} \longrightarrow \mathcal{E}_{i}^{+} \longrightarrow \ldots \longrightarrow \varepsilon_{0}^{+}=\varepsilon,
$$

where nonlocal variables in $\mathcal{E}_{i}^{+}$are $q_{\alpha, x}^{k}, 1 \leq \alpha \leq i, k \geq 0$.

Proposition 1. The 2-forms

$$
\omega_{i+1}^{k}=\left(\tilde{D}_{x}^{k}\left(q_{i+1, y}\right) d y+\tilde{D}_{x}^{k}\left(q_{i+1, t}\right) d t\right) \wedge d x
$$

are linearly independent 2-component conservation laws on $\mathcal{E}_{i}^{+}$.

When proving this statement, as well as Proposition 2, we use the following fact (see [14]): Let $\mathcal{E}$ be a differentially connected equation (i.e., an equation such that the only functions on $\mathcal{E}$ annihilated by all total derivatives are constants) and let $\tau_{\Omega}: \mathcal{E}_{\Omega} \rightarrow \mathcal{E}$ be an Abelian covering associated with a system of conservation laws $\Omega=\left\{\omega_{1}, \ldots, \omega_{s}\right\}$. Then these conservation laws are linearly independent if and only if the covering equation is differentially connected as well.

Proof. The proof uses a double induction: on $i$ and on $k$ for each $i$. Obviously, VWE is differentially connected; denote by $\mathcal{K}_{s}$ the space of functions that are annihilated by $\tilde{D}_{x}, \tilde{D}_{y}, \tilde{D}_{t}$ and have jet order $\leq s$. Let $f \in \mathcal{K}_{s}$.

Step $1(i=1, k=0)$. We have

$$
\tilde{D}_{y}=D_{y}+\frac{1}{\lambda} \frac{u_{y}}{u_{x}} \frac{\partial}{\partial q_{1}}
$$

in this case, from where it follows that $s=0$, i.e., $f=f\left(x, y, t, u, q_{1}\right)$. Hence, $f$ must be invariant w.r.t.

$$
\frac{\partial}{\partial y}+u_{y} \frac{\partial}{\partial u}+\frac{1}{\lambda} \frac{u_{y}}{u_{x}} \frac{\partial}{\partial q_{1}}
$$

But $f$ is independent of $u_{x}$; consequently, it does not depend on $q_{1}$.

Step $2(i=1, k>0)$. One has

$$
\tilde{D}_{y}=D_{y}+\frac{1}{\lambda} \sum_{\alpha=0}^{k} \tilde{D}_{x}^{\alpha}\left(\frac{u_{y}}{u_{x}}\right) \frac{\partial}{\partial q_{1, x}^{\alpha}} .
$$

The maximal jet order of the nonlocal summand is $k+1$ and the variables of this order are $u_{x^{k+1}}$ and $u_{x^{k} y}$. Using expression (4) for $\tilde{D}_{y}$, we see that

$$
Z_{1}=\left[\frac{\partial}{\partial u_{x^{k+1}}}, \tilde{D}_{y}\right]=-\frac{\lambda+1}{\lambda} \frac{u_{t} u_{x y}-u_{y} u_{x t}}{u_{x}^{2}} \frac{\partial}{\partial u_{x^{k} t}}-\frac{1}{\lambda} \frac{u_{y}}{u_{x}^{2}} \frac{\partial}{\partial q_{1, x}^{k}} .
$$


On the other hand, since the coefficients of $\tilde{D}_{y}$ do not depend on $u_{y^{l}}, l>1$, the function $f \in \mathcal{K}_{s}$ cannot depend on $u_{y^{l}}, l>0$. Consequently, it must be invariant w.r.t.

$$
Z_{2}=\left[\frac{\partial}{\partial u_{y}}, Z_{1}\right]=\frac{\lambda+1}{\lambda} \frac{u_{x t}}{u_{x}^{2}} \frac{\partial}{\partial u_{x^{k} t}}-\frac{1}{\lambda} \frac{1}{u_{x}^{2}} \frac{\partial}{\partial q_{1, x}^{k}} .
$$

But the field $\partial / \partial q_{1, x}^{k}$ is a linear combination of $Z_{1}$ and $Z_{2}$; hence, $f$ does not depend on $q_{1, x}^{k}$. Step $3(i>1, k=0)$. From Equations (8) and (7) we see that

$$
\begin{aligned}
\tilde{D}_{y}=D_{y}+\frac{1}{\lambda} \sum_{\beta=1}^{i} \sum_{\alpha=0}^{k_{\beta}} \tilde{D}_{x}^{\alpha}\left(\frac{u_{y} q_{\beta-1, x}}{u_{x}}\right) \frac{\partial}{\partial q_{\beta, x}^{\alpha}} & \\
& =D_{y}+\frac{1}{\lambda} \sum_{\beta=1}^{i} \sum_{\alpha=0}^{k_{\beta}}\left(\frac{u_{y}}{u_{x}} q_{\beta-1, x}^{\alpha+1}+\alpha \tilde{D}_{x}\left(\frac{u_{y}}{u_{x}}\right) q_{\beta-1, x}^{\alpha}+\ldots\right) \frac{\partial}{\partial q_{\beta, x}^{\alpha}}
\end{aligned}
$$

This means that the inequalities

$$
k_{1}>k_{2}>\cdots>k_{i}
$$

hold. Consequently, the maximal jet order of the coefficients appears at the term

$$
\frac{1}{\lambda} \tilde{D}_{x}^{k_{1}}\left(\frac{u_{y}}{u_{x}} q_{1, x}\right) \frac{\partial}{\partial q_{1, x}^{k_{1}}},
$$

which means that repeating the reasoning of Step 2 one can prove that $f \in \mathcal{K}_{s}$ is independent of the variables $q_{1, x}^{k_{1}}, \ldots, q_{1, x}^{k_{2}+1}$, i.e., $k_{1}=k_{2}$, which is impossible by (9).

Step $4(i>1, k>0)$. The proof at this step repeats literary the one accomplished at Step 2 for $i=1$.

The result is proved.

1.2. The negative covering. The negative covering $\tau^{-}: \mathcal{E}^{-} \rightarrow \mathcal{E}$ is defined by the system

$$
r_{i, t}=\frac{(\lambda+1) u_{t} r_{i-1, y}}{u_{y}}-r_{i-1, t}, \quad r_{i, x}=\frac{\lambda u_{x} r_{i-1, y}}{u_{y}}
$$

where $i \geq 1$ and $r_{0}=y$. Nonlocal variables in $\mathcal{E}^{-}$are $r_{i, y}^{k}, i \geq 1, k \geq 0$ (in particular, $r_{i, x}^{0}=r_{i}$ ), while the total derivatives take the form

$$
\tilde{D}_{x}=D_{x}+\sum_{i, k} \tilde{D}_{y}^{k}\left(r_{i, x}\right) \frac{\partial}{\partial r_{i, y}^{k}}, \quad \tilde{D}_{y}=D_{y}+\sum_{i, k} r_{i, y}^{k+1} \frac{\partial}{\partial r_{i, y}^{k}}, \quad \tilde{D}_{t}=D_{t}+\sum_{i, k} \tilde{D}_{y}^{k}\left(r_{i, t}\right) \frac{\partial}{\partial r_{i, y}^{k}},
$$

where $r_{i, x}, r_{i, t}$ are given by Equations (10).

Similar to the positive case, we consider the tower

$$
\mathcal{E}^{-} \longrightarrow \ldots \longrightarrow \mathcal{E}_{i-1}^{-} \longrightarrow \mathcal{E}_{i}^{-} \longrightarrow \ldots \longrightarrow \mathcal{E}_{0}^{-}=\mathcal{E}
$$

where nonlocal variables in $\mathcal{E}_{i}^{-}$are $r_{\alpha, y}^{k}, 1 \leq \alpha \leq i, k \geq 0$.

Proposition 2. The 2-forms

$$
\theta_{i+1}^{k}=\left(\tilde{D}_{y}^{k}\left(r_{i+1, x}\right) d x+\tilde{D}_{y}^{k}\left(r_{i+1, t}\right) d t\right) \wedge d y
$$

are linearly independent 2-component conservation laws on $\mathcal{E}_{i}^{-}$.

Proof. The proof of this statement is similar to the one of Proposition 1, but we must work with the field $\tilde{D}_{x}$ instead of $\tilde{D}_{y}$. 


\section{LIE ALGEBRAS OF NONLOCAL SYMMETRIES}

Local symmetries of $\mathcal{E}$ are solutions to the linearization

$$
\ell_{\varepsilon}(\varphi)=0
$$

of Equation (11), where

$$
\ell_{\varepsilon}=\left(u_{y} D_{x} D_{t}+u_{x t} D_{y}\right)+\lambda\left(u_{x} D_{t} D_{y}+u_{t y} D_{x}\right)-(\lambda+1)\left(u_{t} D_{x} D_{y}+u_{x y} D_{t}\right)
$$

they form a Lie algebra w.r.t. the Jacobi bracket $\{\cdot, \cdot\}$ denoted by $\operatorname{sym}(\mathcal{E})$. The corresponding vector field on $\mathcal{E}$ is the evolution derivation

$$
\mathbf{E}_{\varphi}=\sum D_{\sigma}(\varphi) \frac{\partial}{\partial u_{\sigma}}
$$

where summation is done over all the internal coordinates $u_{\sigma}$ on $\mathcal{E}$.

Direct computations lead to the following

Proposition 3. The algebra $\operatorname{sym}(\mathcal{E})$ is spanned by the elements

$$
\varphi_{1}(T)=T u_{t}, \quad \varphi_{2}(X)=X u_{x}, \quad \varphi_{3}(Y)=Y u_{y}, \quad \varphi_{4}(U)=U
$$

where $T=T(t), X=X(x), Y=Y(y), U=U(u)$ are arbitrary smooth functions. The non-zero commutators are

$$
\begin{aligned}
& \left\{\varphi_{1}(T), \varphi_{1}(\tilde{T})\right\}=\varphi_{1}([\tilde{T}, T]), \\
& \left\{\varphi_{2}(X), \varphi_{2}(\tilde{X})\right\}=\varphi_{2}([\tilde{X}, X]), \\
& \left\{\varphi_{3}(Y), \varphi_{3}(\tilde{Y})\right\}=\varphi_{3}([\tilde{Y}, Y]), \\
& \left\{\varphi_{4}(U), \varphi_{4}(\tilde{U})\right\}=\varphi_{4}([U, \tilde{U}]),
\end{aligned}
$$

where $[Z, \tilde{Z}]$ denotes $Z \partial \tilde{Z} / \partial z-\tilde{Z} \partial Z / \partial z$ for any functions $Z$ and $\tilde{Z}$ in $z$.

2.1. The algebra $\operatorname{sym}_{\tau^{+}}(\mathcal{E})$. To find the Lie algebra $\operatorname{sym}_{\tau^{+}}(\mathcal{E})$ of nonlocal symmetries in the positive covering $\tau^{+}$, one needs to solve the following system:

$$
\begin{aligned}
\tilde{\ell}_{\varepsilon}(\varphi) & =0 \\
\tilde{D}_{t}\left(\varphi^{i}\right) & =\frac{(\lambda+1)}{\lambda}\left(\frac{u_{x} \tilde{D}_{t}(\varphi)-u_{t} \tilde{D}_{x}(\varphi)}{u_{x}^{2}} q_{i-1, x}+\frac{u_{t}}{u_{x}} \tilde{D}_{x}\left(\varphi^{i-1}\right)\right)-\tilde{D}_{t}\left(\varphi^{i-1}\right), \\
\tilde{D}_{y}\left(\varphi^{i}\right) & =\frac{1}{\lambda}\left(\frac{u_{x} \tilde{D}_{y}(\varphi)-u_{y} \tilde{D}_{x}(\varphi)}{u_{x}^{2}} q_{i-1, x}+\frac{u_{y}}{u_{x}} \tilde{D}_{x}\left(\varphi^{i-1}\right)\right)
\end{aligned}
$$

where $\tilde{\ell}_{\varepsilon}$ denotes the natural lift of the operator (13) from $\mathcal{E}$ to $\mathcal{E}^{+}$. Solutions of (14) are denoted by

$$
\Phi=\left[\varphi, \varphi^{1}, \ldots, \varphi^{i}, \ldots\right]
$$

and to any such a $\Phi$ there corresponds the vector field

$$
S_{\Phi}=\tilde{\mathbf{E}}_{\varphi}+\sum_{i, k} \tilde{D}_{x}^{k}\left(\varphi^{i}\right) \frac{\partial}{\partial q_{i, x}^{k}}
$$

on $\mathcal{E}^{+}$. Solutions of the first equation in (14) are called nonlocal $\tau^{+}$-shadows. In particular, local symmetries can be considered as shadows in any covering. If $\varphi$ is a shadow and there exists a nonlocal symmetry $\Phi=\left[\varphi, \varphi^{1}, \ldots\right]$ then we say that this shadow lifts to the covering. Nonlocal symmetries $\Phi$ with $\varphi=0$ (i.e., with trivial shadows) are called invisible. Given $\Phi$ and $\tilde{\Phi}$, one can define their bracket by

$$
\{\Phi, \tilde{\Phi}\}=S_{\Phi}(\tilde{\Phi})-S_{\tilde{\Phi}}(\Phi),
$$

where the action is component-wise.

Consider the vector field

$$
X=\sum_{i=0}^{\infty}(i+1) q_{i+1} \frac{\partial}{\partial q_{i}},
$$

(recall that $q_{0}=x$ ) and set

$$
P_{0}(X)=X, \quad P_{j}(X)=\frac{1}{j} X\left(P_{j-1}(X)\right), \quad j \geq 1 .
$$

Proposition 4. All the local symmetries of the VWE can be lifted to the positive covering. 
Proof. Denote by $\Phi_{\alpha}=\left[\varphi_{\alpha}, \varphi_{\alpha}^{1}, \ldots, \varphi_{\alpha}^{i}, \ldots\right], \alpha=1, \ldots, 4$, the lifts to be constructed and set

$$
\varphi_{1}^{i}(T)=T q_{i, t}, \quad \varphi_{2}^{i}(X)=X q_{i, x}-P_{i}(X), \quad \varphi_{3}^{i}(Y)=Y q_{i, y}, \quad \varphi_{4}^{i}(U)=0 .
$$

A direct computation shows that the functions $\Phi_{1}=\Phi_{1}(T), \Phi_{2}=\Phi_{2}(X), \Phi_{3}=\Phi_{3}(Y)$, and $\Phi_{4}=$ $\Phi_{4}(U)$ are the desired lifts.

We now construct two series of $\tau^{+}$-nonlocal symmetries.

The first one, denoted by $\Psi_{k}^{+}=\left[\psi_{k}^{+}, \psi_{k}^{+, 1}, \ldots, \psi_{k}^{+, i}, \ldots\right], k \geq 2$, arises as follows. The symmetries $\Psi_{2}^{+}, \Psi_{3}^{+}$, and $\Psi_{4}^{+}$are introduced "by hand":

$$
\begin{aligned}
\psi_{2}^{+} & =\left(2 q_{2}-q_{1}\left(q_{1, x}-1\right)\right) u_{x}, \\
\psi_{2}^{+, i} & =-(i+2) q_{i+2}-(i+1) q_{i+1}+2 q_{2} q_{i, x}+q_{1}\left(q_{i+1, x}-\left(q_{1, x}-1\right) q_{i, x}\right) \\
& +\frac{1}{\lambda}\left((i+1) q_{i+1}+i q_{i}-q_{1} q_{i, x}\right) ; \\
\psi_{3}^{+} & =\left(3 q_{3}-2 q_{2} q_{1, x}-q_{1}\left(q_{2, x}-q_{1, x}^{2}+1\right)\right) u_{x}, \\
\psi_{3}^{+, i} & =-(i+3) q_{i+3}+(i+1) q_{i+1}+3 q_{3} q_{i, x}+2 q_{2}\left(q_{i+1, x}-q_{1, x} q_{i, x}\right) \\
& +q_{1}\left(q_{i+2, x}-q_{1, x} q_{i+1, x}-\left(q_{2, x}-q_{1, x}^{2}+1\right) q_{i, x}\right) \\
& +\frac{1}{\lambda}\left((i+2) q_{i+2}-i q_{i}-2 q_{2} q_{i, x}-q_{1}\left(q_{i+1, x}-q_{1, x} q_{i, x}\right)\right) ; \\
\psi_{4}^{+} & =\left(4 q_{4}-3 q_{3} q_{1, x}-2 q_{2}\left(q_{2, x}-q_{1, x}^{2}\right)-q_{1}\left(q_{3, x}-2 q_{1, x} q_{2, x}+q_{1, x}^{3}-1\right)\right) u_{x}, \\
\psi_{4}^{+, i} & =-(i+4) q_{i+4}-(i+1) q_{i+1}+4 q_{4} q_{i, x}+3 q_{3}\left(q_{i+1, x}-q_{1, x} q_{i, x}\right) \\
& +2 q_{2}\left(q_{i+2, x}-q_{1, x} q_{i+1, x}-\left(q_{2, x}-q_{1, x}^{2}\right) q_{i, x}\right) \\
& +q_{1}\left(q_{i+3, x}-q_{1, x} q_{i+2, x}-\left(q_{2, x}-q_{1, x}^{2}\right) q_{i+1, x}-\left(q_{3, x}-2 q_{1, x} q_{2, x}+q_{1, x}^{3}-1\right) q_{i, x}\right) \\
& +\frac{1}{\lambda}\left((i+3) q_{i+3}+i q_{i}-3 q_{3} q_{i, x}-2 q_{2}\left(q_{i+1, x}-q_{1, x} q_{i, x}\right)\right. \\
& \left.-q_{1}\left(q_{i+2, x}-q_{1, x} q_{i+1, x}-\left(q_{2, x}-q_{1, x}^{2}\right) q_{i, x}\right)\right) .
\end{aligned}
$$

For $k>4$, we set

$$
\begin{aligned}
\Psi_{k}^{+}=\frac{1}{k-4}\left(\left\{\Psi_{k-2}^{+}, \Psi_{2}^{+}\right\}-(k-3) \Psi_{k-1}^{+}+(-1)^{k} \Psi_{3}^{+}\right. & \\
& \left.+\frac{1}{\lambda}\left((k-4) \Psi_{k-1}^{+}+(k-3) \Psi_{k-2}^{+}-(-1)^{k} \Psi_{2}^{+}\right)\right) .
\end{aligned}
$$

Now, the second series $\Xi_{k}^{+}(X)=\left[\xi_{k}^{+}(X), \xi_{k}^{+, 1}(X), \ldots, \xi_{k}^{+, i}(X), \ldots\right], k \geq 1$, is defined by the relations

$$
\begin{aligned}
\xi_{1}^{+}(X) & =\left(X q_{1, x}-X_{x} q_{1}\right) u_{x}, \\
\xi_{1}^{+, i}(X) & =X\left(q_{1, x} q_{i, x}-q_{i+1, x}\right)-X_{x} q_{1} q_{i, x}+P_{i+1}(X)+\frac{1}{\lambda}\left(X q_{i, x}-P_{i}(X)\right) ; \\
\xi_{2}^{+}(X) & =\left(X\left(q_{2, x}-q_{1, x}^{2}\right)+X_{x}\left(q_{1} q_{1, x}-q_{2}\right)-\frac{1}{2} X_{x x} q_{1}^{2}\right) u_{x}, \\
\xi_{2}^{+, i}(X) & =X\left(q_{1, x} q_{i+1, x}+\left(q_{2, x}-q_{1, x}^{2}\right) q_{i, x}-q_{i+2, x}\right)-X_{x}\left(q_{2} q_{i, x}+q_{1}\left(q_{i+1, x}-q_{1, x} q_{i, x}\right)\right) \\
& -\frac{1}{2} X_{x x} q_{1}^{2} q_{i, x}+P_{i+2}(X)+\frac{1}{\lambda}\left(X\left(q_{i+1, x}-q_{1, x} q_{i, x}\right)+X_{x} q_{1} q_{i, x}-P_{i+1}(X)\right),
\end{aligned}
$$

where the functions $P_{i}(X)$ are given by relations (16), and

$$
\Xi_{k}^{+}(X)=\frac{1}{k-2}\left(\left\{\Xi_{k-2}^{+}(X), \Psi_{2}^{+}\right\}-\Xi_{k-1}^{+}(X)+\frac{1}{\lambda}(k-3)\left(\Xi_{k-1}^{+}(X)+\Xi_{k-2}^{+}(X)\right)\right)
$$

for $k \geq 3$.

Finally, invisible symmetries in $\tau^{+}$are

$$
\Phi_{k}^{\mathrm{inv}}(X)=[\underbrace{0, \ldots, 0}_{k-\text { times }}, P_{0}(X), P_{1}(X), \ldots]
$$


where $k \geq 1$ and $P_{i}(X)$ are given by (16), as above.

To describe the Lie algebra structure in $\operatorname{sym}_{\tau^{+}}(\mathcal{E})$, it is convenient to relabel the above introduced symmetries. Namely, we change the generators of $\operatorname{sym}_{\tau^{+}}(\mathcal{E})$ as follows:

$$
\Phi_{2}(X) \mapsto-\Xi_{0}^{+}(X), \quad \Phi_{k}^{\mathrm{inv}}(X) \mapsto \Xi_{-k}^{+}(X), k \geq 1,
$$

and

$$
\Psi_{k}^{+} \mapsto(-1)^{k+1} \Psi_{k}^{+}, k \geq 2, \quad \Xi_{k}^{+}(X) \mapsto(-1)^{k} \Xi_{k}^{+}(X), k \in \mathbb{Z} .
$$

Proposition 5. In the new basis, the Lie algebra structure of $\operatorname{sym}_{\tau^{+}}(\mathcal{E})$ is given by the brackets

$$
\left\{\Psi_{i}^{+}, \Psi_{j}^{+}\right\}=(j-i)\left(\Psi_{i+j}^{+}+\frac{1}{\lambda} \Psi_{i+j-1}^{+}\right)-(j-1)\left(\Psi_{j+1}^{+}+\frac{1}{\lambda} \Psi_{j}^{+}\right)+(i-1)\left(\Psi_{i+1}^{+}+\frac{1}{\lambda} \Psi_{i}^{+}\right)
$$

for all $j>i \geq 2$. One also has

$$
\left\{\Xi_{i}^{+}(X), \Xi_{j}^{+}(\tilde{X})\right\}= \begin{cases}\Xi_{i+j}^{+}([X, \tilde{X}]), & i, j \leq 0 \text { or } i<0, j>0, i+j>0, \\ \Xi_{i+j}^{+}([X, \tilde{X}])+\frac{1}{\lambda} \Xi_{i+j-1}^{+}([X, \tilde{X}]), & \text { otherwise, }\end{cases}
$$

$i, j \in \mathbb{Z}$, and

$$
\left\{\Psi_{i}^{+}, \Xi_{j}^{+}(X)\right\}= \begin{cases}j\left(\Xi_{i+j}^{+}(X)-\Xi_{j+1}^{+}(X)\right)+\frac{1}{\lambda}\left((j-1) \Xi_{i+j-1}^{+}(X)-\Xi_{j}^{+}(X)\right), & j \geq 1, \\ j\left(\Xi_{i+j}^{+}(X)-\Xi_{j+1}^{+}(X)-\frac{1}{\lambda} \Xi_{j}^{+}(x)\right), & j<1, i+j>0, \\ j\left(\Xi_{i+j}^{+}(X)-\Xi_{j+1}^{+}(X)-\frac{1}{\lambda}\left(\Xi_{i+j-1}^{+}(X)-\Xi_{j}^{+}(X)\right)\right), & \text { otherwise, }\end{cases}
$$

$i \geq 2, j \in \mathbb{Z}$. All the other commutators vanish.

2.2. The algebra $\operatorname{sym}_{\tau^{-}}(\mathcal{E})$. Computations here go along the same lines as in Subsection 2.1 and we use similar notation below. The defining equations are

$$
\begin{aligned}
\tilde{\ell}_{\varepsilon}(\varphi) & =0 \\
\tilde{D}_{t}\left(\varphi^{i}\right) & =(\lambda+1)\left(\frac{u_{y} \tilde{D}_{t}(\varphi)-u_{t} \tilde{D}_{y}(\varphi)}{u_{y}^{2}} r_{i-1, y}+\frac{u_{t}}{u_{y}} \tilde{D}_{y}\left(\varphi^{i-1}\right)\right)-\tilde{D}_{t}\left(\varphi^{i-1}\right), \\
\tilde{D}_{x}\left(\varphi^{i}\right) & =\lambda\left(\frac{u_{y} \tilde{D}_{x}(\varphi)-u_{x} \tilde{D}_{y}(\varphi)}{u_{y}^{2}} r_{i-1, y}+\frac{u_{x}}{u_{y}} \tilde{D}_{y}\left(\varphi^{i-1}\right)\right),
\end{aligned}
$$

where "tilde" marks operators on $\mathcal{E}^{-}$. A solution $\Phi=\left[\varphi, \varphi^{1}, \ldots, \varphi^{i}, \ldots\right]$ of (17) corresponds to the vector field

$$
S_{\Phi}=\tilde{\mathbf{E}}_{\Phi}+\sum_{i, k} \tilde{D}_{y}^{k}\left(\varphi^{i}\right) \frac{\partial}{\partial r_{i, y}^{k}}
$$

on $\mathcal{E}^{-}$and the bracket $\{\Phi, \tilde{\Phi}\}=S_{\Phi}(\tilde{\Phi})-S_{\tilde{\Phi}}(\Phi)$ is defined for such solutions.

To proceed with further constructions, we will introduce the vector field

$$
y=\sum_{i=0}^{\infty}(i+1) r_{i+1} \frac{\partial}{\partial r_{i}}
$$

and the quantities $Q_{j}, j=0,1 \ldots$ defined as follows:

$$
Q_{0}(Y)=Y, \quad Q_{j}(Y)=\frac{1}{j} y\left(Q_{j-1}(Y)\right), \quad j \geq 1,
$$

(recall that $\left.r_{0}=y\right)$.

Proposition 6. All the local symmetries of the WVE can be lifted to the negative covering.

Proof. Denote the lifts by $\Phi_{\alpha}=\left[\varphi_{\alpha}, \varphi_{\alpha}^{1}, \ldots, \varphi_{\alpha}^{i}, \ldots\right], \alpha=1, \ldots, 4$, and set

$$
\varphi_{1}^{i}(T)=T r_{i, t}, \quad \varphi_{2}^{i}(X)=X r_{i, x}, \quad \varphi_{3}^{i}(Y)=Y r_{i, y}-Q_{i}(Y), \quad \varphi_{4}^{i}(U)=0 .
$$

The rest of proof is a straightforward check. 
Let us now construct, similar to the positive case, two series of nonlocal symmetries. The first one $\Psi_{k}^{-}=\left[\psi_{k}^{-}, \psi_{k}^{-, 1}, \ldots, \psi_{k}^{-, i}, \ldots\right], k \geq 2$, is defined as follows. For $k=2,3,4$ we set

$$
\begin{aligned}
\psi_{2}^{-} & =\left(2 r_{2}-r_{1}\left(r_{1, y}-1\right)\right) u_{y} \\
\psi_{2}^{-, i} & =-(i+2) r_{i+2}-(i+1) r_{i+1}+2 r_{2} r_{i, y}+r_{1}\left(r_{i+1, y}-\left(r_{1, y}-1\right) r_{i, y}\right) \\
& +\lambda\left((i+1) r_{i+1}+i r_{i}-r_{1} r_{i, y}\right) \\
\psi_{3}^{-} & =\left(3 r_{3}-2 r_{2} r_{1, y}-r_{1}\left(r_{2, y}-r_{1, y}^{2}+1\right)\right) u_{y}, \\
\psi_{3}^{-, i} & =-(i+3) r_{i+3}+(i+1) r_{i+1}+3 r_{3} r_{i, y}+2 r_{2}\left(r_{i+1, y}-r_{1, y} r_{i, y}\right) \\
& +r_{1}\left(r_{i+2, y}-r_{1, y} r_{i+1, y}-\left(r_{2, y}-r_{1, y}^{2}+1\right) r_{i, y}\right) \\
& +\lambda\left((i+2) r_{i+2}-i r_{i}-2 r_{2} r_{i, y}-r_{1}\left(r_{i+1, y}-r_{1, y} r_{i, y}\right)\right) \\
\psi_{4}^{-} & =\left(4 r_{4}-3 r_{3} r_{1, y}-2 r_{2}\left(r_{2, y}-r_{1, y}^{2}\right)-r_{1}\left(r_{3, y}-2 r_{1, y} r_{2, y}+r_{1, y}^{3}-1\right)\right) u_{y}, \\
\psi_{4}^{-, i} & =-(i+4) r_{i+4}-(i+1) r_{i+1}+4 r_{4} r_{i, y}+3 r_{3}\left(r_{i+1, y}-r_{1, y} r_{i, y}\right) \\
& +2 r_{2}\left(r_{i+2, y}-r_{1, y} r_{i+1, y}-\left(r_{2, y}-r_{1, y}^{2}\right) r_{i, y}\right) \\
& +r_{1}\left(r_{i+3, y}-r_{1, y} r_{i+2, y}-\left(r_{2, y}-r_{1, y}^{2}\right) r_{i+1, y}-\left(r_{3, y}-2 r_{1, y} r_{2, y}+r_{1, y}^{3}-1\right) r_{i, y}\right) \\
& +\lambda\left((i+3) r_{i+3}+i r_{i}-3 r_{3} r_{i, y}-2 r_{2}\left(r_{i+1, y}-r_{1, y} r_{i, y}\right)-r_{1}\left(r_{i+2, y}-r_{1, y} r_{i+1, y}-\left(r_{2, y}-r_{1, y}^{2}\right) r_{i, y}\right)\right) .
\end{aligned}
$$

For $k>4$ we define

$$
\begin{aligned}
\Psi_{k}^{-}=\frac{1}{k-4}\left(\left\{\Psi_{k-2}^{-}, \Psi_{2}^{-}\right\}-(k-3) \Psi_{k-1}^{-}+(-1)^{k} \Psi_{3}^{-}\right. & \\
& \left.+\lambda\left((k-4) \Psi_{k-1}^{-}+(k-3) \Psi_{k-2}^{-}-(-1)^{k} \Psi_{2}^{-}\right)\right) .
\end{aligned}
$$

Introduce the second series $\Xi_{k}^{-}(Y)=\left[\xi_{k}^{-}(Y), \xi_{k}^{-, 1}(Y), \ldots, \xi_{k}^{-, i}(Y), \ldots\right]$ now by

$$
\begin{aligned}
\xi_{1}^{-}(Y) & =\left(Y r_{1, y}-Y_{y} r_{1}\right) u_{y}, \\
\xi_{1}^{-, i}(Y) & =Y\left(r_{1, y} r_{i, y}-r_{i+1, y}\right)-Y_{y} r_{1} r_{i, y}+Q_{i+1}(Y)+\lambda\left(Y r_{i, y}-Q_{i}(Y)\right) ; \\
\xi_{2}^{-}(Y) & =\left(Y\left(r_{2, y}-r_{1, y}^{2}\right)+Y_{y}\left(r_{1} r_{1, y}-r_{2}\right)-\frac{1}{2} Y_{y y} r_{1}^{2}\right) u_{y}, \\
\xi_{2}^{-, i}(Y) & =Y\left(r_{1, y} r_{i+1, y}+\left(r_{2, y}-r_{1, y}^{2}\right) r_{i, y}-r_{i+2, y}\right)-Y_{y}\left(r_{2} r_{i, y}+r_{1}\left(r_{i+1, y}-r_{1, y} r_{i, y}\right)\right) \\
& -\frac{1}{2} Y_{y y} r_{1}^{2} r_{i, y}+Q_{i+2}(Y)+\lambda\left(Y\left(r_{i+1, y}-r_{1, y} r_{i, y}\right)+Y_{y} r_{1} r_{i, y}-Q_{i+1}(Y)\right)
\end{aligned}
$$

and for $k \geq 3$

$$
\Xi_{k}^{-}(Y)=\frac{1}{k-2}\left(\left\{\Xi_{k-2}^{-}(Y), \Psi_{2}\right\}-\Xi_{k-1}^{-}(Y)+\lambda(k-3)\left(\Xi_{k-1}^{-}(Y)+\Xi_{k-2}^{-}(Y)\right)\right) .
$$

Invisible symmetries in $\tau^{-}$have the form

$$
\Phi_{k}^{\mathrm{inv}}(Y)=[\underbrace{0, \ldots, 0}_{k-\text { times }}, Q_{0}(Y), Q_{1}(Y), \ldots]
$$

where $k \geq 1$ and $Q_{i}(Y)$ are given by (19).

We again relabel the generators by

$$
\Phi_{3}(Y) \mapsto-\Xi_{0}^{-}(Y), \quad \Phi_{k}^{\mathrm{inv}}(Y) \mapsto \Xi_{-k}^{-}(Y), k \geq 1,
$$

and

$$
\Psi_{k}^{-} \mapsto(-1)^{k+1} \Psi_{k}^{-}, k \geq 2, \quad \Xi_{k}^{-}(Y) \mapsto(-1)^{k} \Xi_{k}^{-}(Y), k \in \mathbb{Z} .
$$

Then the following statement holds:

Proposition 7. The above defined generators enjoy the following relations:

$$
\left\{\Psi_{i}^{-}, \Psi_{j}^{-}\right\}=(j-i)\left(\Psi_{i+j}^{-}+\lambda \Psi_{i+j-1}^{-}\right)-(j-1)\left(\Psi_{j+1}^{-}+\lambda \Psi_{j}^{-}\right)+(i-1)\left(\Psi_{i+1}^{-}+\lambda \Psi_{i}^{-}\right)
$$


for $j>i \geq 2$,

$$
\left\{\Xi_{i}^{-}(Y), \Xi_{j}^{-}(\tilde{Y})\right\}= \begin{cases}\Xi_{i+j}^{-}([Y, \tilde{Y}]), & i \leq 0, j>0, i+j>0 \text { or } j \leq 0, \\ \Xi_{i+j}^{-}([Y, \tilde{Y}])+\lambda \Xi_{i+j-1}^{-}([Y, \tilde{Y}]), & \text { otherwise, }\end{cases}
$$

for all $i<j \in \mathbb{Z}$, and

$$
\left\{\Psi_{i}^{-}, \Xi_{j}^{-}(Y)\right\}= \begin{cases}j\left(\Xi_{i+j}^{-}(Y)-\Xi_{j+1}^{-}(Y)\right)+\lambda\left((j-1) \Xi_{i+j-1}^{-}(Y)-\Xi_{j}^{-}(Y)\right), & j \geq 1 \\ j\left(\Xi_{i+j}^{-}(Y)-\Xi_{j+1}^{-}(Y)-\lambda \Xi_{j}^{-}(Y)\right), & j<1, i+j>0, \\ j\left(\Xi_{i+j}^{-}(Y)-\Xi_{j+1}^{-}(Y)+\lambda\left(\Xi_{i+j-1}^{-}(Y)-\Xi_{j}^{-}(Y)\right)\right), & \text { otherwise, }\end{cases}
$$

for $j \in \mathbb{Z}, i \geq 2$.

\section{RECURSION OPERATORS AND A MASTER-SYMMETRY}

According to the general theory, see [21], recursion operators for symmetries arise as Bäcklund auto-transformations of the tangent space $\mathcal{T E}$, cf. [15]. In the case of VWE, this BT is

$$
\begin{aligned}
& u_{x} \tilde{D}_{t}(\zeta)=u_{t x} \zeta-u_{x} \tilde{D}_{t}(\eta)+\frac{\lambda+1}{\lambda} u_{t} \tilde{D}_{x}(\eta)-\frac{1}{\lambda} u_{t x} \eta, \\
& u_{x} \tilde{D}_{y}(\zeta)=u_{x y} \zeta+\frac{1}{\lambda} u_{y} \tilde{D}_{x}(\eta)-\frac{1}{\lambda} u_{x y} \eta .
\end{aligned}
$$

Proposition 8. Let $\eta$ be a $\tau^{ \pm}$-shadow. Then $\zeta=\mathcal{R}_{+}(\eta)$ obtained as a solution of (20) is a shadow as well. Vice versa, if $\eta$ is a shadow the $\zeta=\mathcal{R}_{-}(\eta)$ obtained in the same way is a shadow too.

Proof. To construct a recursion operator for Equation (1) we use the techniques of [25], cf. [20, 22, 24, [17, 4] also. We find a shadow for VWE in the covering (5). It is of the form $s=H(w) u_{x} w_{x}^{-1}$, where $H$ is an arbitrary function in $w$. Since System (5) is invariant with respect to the transformation $w \mapsto$ $H(w)$, we put, without loss of generality, $s=u_{x} w_{x}^{-1}$. Differentiation of (5) by $x$ and substitution $q_{x}=u_{x} s^{-1}$ gives another covering

$$
s_{t}=\frac{\mu(\lambda+1) u_{t}}{\lambda(\mu+1) u_{x}} s_{x}+\frac{(\lambda-\mu) u_{t x}}{\lambda u_{x}} s, \quad s_{y}=\frac{\mu u_{y}}{\lambda u_{x}} s_{x}+\frac{(\lambda-\mu) u_{x y}}{\lambda u_{x}} s
$$

for Equation (11). Note that $s$ is a solution to the linearization (12), (13) of VWE. Now put

$$
s=\sum_{n=-\infty}^{\infty} s_{n} \mu^{n}
$$

Since system (12), (13) is independent of $\mu$, each $s_{n}$ is a solution to this system as well. Substituting (22) to system (12), (13) yields

$$
\begin{aligned}
& s_{n+1, t}=\frac{u_{t x}}{u_{x}} s_{n+1}-s_{n, t}+\frac{\lambda+1}{\lambda} \frac{u_{t}}{u_{x}} s_{n, x}-\frac{1}{\lambda} \frac{u_{t x}}{u_{x}} s_{n}, \\
& s_{n+1, y}=\frac{u_{x y}}{u_{x}} s_{n+1}+\frac{1}{\lambda} \frac{u_{y}}{u_{x}} s_{n, x}-\frac{1}{\lambda} \frac{u_{x y}}{u_{x}} s_{n} .
\end{aligned}
$$

Relabeling $s_{n}=\eta$ and $s_{n+1}=\zeta$, we obtain the result.

Thus, $\mathcal{R}_{+}$and $\mathcal{R}_{-}$are mutually inverse recursion operators.

3.1. Action of recursion operators. We now describe the action of the operators $\mathcal{R}_{+}$and $\mathcal{R}_{-}$in detail. First of all, it immediately follows from (20) that

$$
\mathcal{R}_{+}(0)=\xi_{0}^{+}(X), \quad \mathcal{R}_{-}(0)=\xi_{0}^{-}(Y)
$$

and thus all subsequent actions of $\mathcal{R}_{+}$and $\mathcal{R}_{-}$are defined modulo addition of $\xi_{0}^{+}(X)$ and $\xi_{0}^{-}(Y)$, respectively.

Further, one has

$$
\begin{array}{ll}
\mathcal{R}_{+}\left(\varphi_{1}(T)\right)=-\varphi_{1}(T), & \mathcal{R}_{-}\left(\varphi_{1}(T)\right)=-\varphi_{1}(T) \\
\mathcal{R}_{+}\left(\varphi_{4}(U)\right)=\lambda^{-1} \varphi_{4}(U), & \mathcal{R}_{-}\left(\varphi_{4}(U)\right)=\lambda \varphi_{4}(U)
\end{array}
$$


and

Finally,

$$
\begin{array}{llll}
\mathcal{R}_{+}\left(\xi_{i}^{+}(X)\right)=\xi_{i+1}^{+}(X), & i \geq 0, & \mathcal{R}_{+}\left(\xi_{i}^{-}(X)\right)=\xi_{i-1}^{-}(X), i \geq 1, & \mathcal{R}_{+}\left(\xi_{0}^{-}(X)\right)=0 ; \\
\mathcal{R}_{-}\left(\xi_{i}^{-}(X)\right)=\xi_{i+1}^{-}(X), i \geq 0, & \mathcal{R}_{-}\left(\xi_{i}^{+}(X)\right)=\xi_{i-1}^{+}(X), i \geq 1, & \mathcal{R}_{-}\left(\xi_{0}^{+}(X)\right)=0 .
\end{array}
$$

$$
\begin{array}{lll}
\mathcal{R}_{+}\left(\psi_{i}^{+}\right)=\psi_{i+1}^{+}, i \geq 2, & \mathcal{R}_{+}\left(\psi_{i}^{-}\right)=\psi_{i-1}^{-}, i \geq 3, & \mathcal{R}_{+}\left(\psi_{2}^{-}\right)=\psi_{1} ; \\
\mathcal{R}_{-}\left(\psi_{i}^{-}\right)=\psi_{i+1}^{-}, i \geq 2, & \mathcal{R}_{-}\left(\psi_{i}^{+}\right)=\psi_{i-1}^{+}, i \geq 3, & \mathcal{R}_{-}\left(\psi_{2}^{+}\right)=\psi_{1} .
\end{array}
$$

The new shadow that arises in Equations (23) "lives" in the Whitney product of $\tau^{+}$and $\tau^{-}$and has the form

$$
\psi_{1}=\lambda u_{x} q_{1}+u_{y} r_{1}
$$

and will be studied in Subsection 3.2 .

The following diagram illustrates the above described actions:

$$
\begin{aligned}
& \varphi_{1}(T) \stackrel{\mathcal{R}_{+}}{\underset{\mathcal{R}_{-}}{\rightleftarrows}} \varphi_{1}(T) \\
& \varphi_{4}(U) \stackrel{\mathcal{R}_{+}}{\stackrel{\mathcal{R}_{-}}{\rightleftarrows}} \varphi_{4}(U)
\end{aligned}
$$

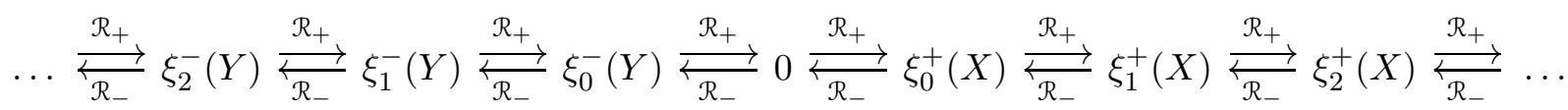

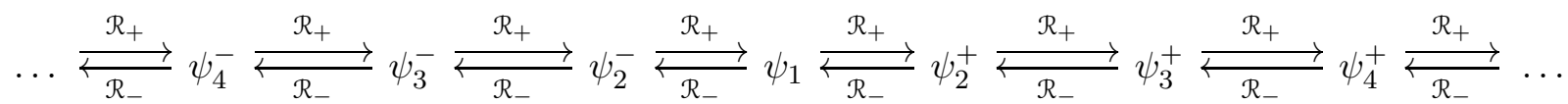

3.2. Master-symmetry. Let us describe the lift

$$
\Psi_{1}=\psi_{1} \frac{\partial}{\partial u}+\sum_{i}\left(\psi_{1}^{+, i} \frac{\partial}{\partial q_{i}}+\psi_{1}^{-, i} \frac{\partial}{\partial r_{i}}\right)
$$

of the shadow $\psi_{1}$ to the Whitney product $\tau^{+} \oplus \tau^{-}$. To this end we set

$$
\begin{aligned}
& \psi_{1}^{+, i}=i q_{i}+(i-1) q_{i-1}+r_{1} q_{i, y}-\lambda\left((i+1) q_{i+1}+i q_{i}-q_{1} q_{i, x}\right), \\
& \psi_{1}^{-, i}=-(i+1) r_{i+1}-i r_{i}+r_{1} r_{i, y}+\lambda\left(i r_{i}+(i-1) r_{i-1}+q_{1} r_{i, x}\right),
\end{aligned}
$$

for all $i \geq 1$, and this is the desired lift. Then the commutators of $\Psi_{1}$ with the already constructed symmetries are as follows:

$$
\left\{\Psi_{1}, \Psi_{i}^{+}\right\}= \begin{cases}\lambda \Psi_{3}^{+}-(2 \lambda-1) \Psi_{2}^{+}-\frac{1}{\lambda} \Psi_{1}, & i=2, \\ \lambda(i-1) \Psi_{i+1}^{+}-((\lambda-1) i+1) \Psi_{i}^{+}-i \Psi_{i-1}^{+}-\frac{1}{\lambda} \Psi_{1}, & i>2,\end{cases}
$$

and

$$
\left\{\Psi_{1}, \Psi_{i}^{-}\right\}= \begin{cases}\Psi_{3}^{-}+(\lambda-2) \Psi_{2}^{-}-\lambda \Psi_{1}, & i=2, \\ (i-1) \Psi_{i+1}^{-}+((\lambda-1) i-\lambda) \Psi_{i}^{-}-\lambda\left(i \Psi_{i-1}^{-}+\Psi_{1}\right), & i>2 .\end{cases}
$$

Further, we have

$$
\left\{\Psi_{1}, \Xi_{i}^{+}(X)\right\}= \begin{cases}i \lambda \Xi_{i+1}^{+}(X)-(i(\lambda-1)+1) \Xi_{i}^{+}(X)-(i-1) \Xi_{i-1}^{+}(X), & i>0, \\ 0, & i=0, \\ i\left(\lambda \Xi_{i+1}^{+}(X)-(\lambda-1) \Xi_{i}^{+}-\Xi_{i-1}^{+}(X)\right), & i \leq-1,\end{cases}
$$

and

$$
\left\{\Psi_{1}, \Xi_{i}^{-}(Y)\right\}= \begin{cases}\left.i \Xi_{i+1}^{-}(Y)+(i(\lambda-1)-\lambda) \Xi_{i}^{-}(Y)-\lambda(i-1) \Xi_{i-1}^{-}(Y)\right), & i>0, \\ 0, & i=0, \\ (i-1)\left(\Xi_{i-1}^{-}(Y)+(\lambda-1) \Xi_{i}^{-}(Y)-\lambda \Xi_{i+1}^{-}(Y)\right), & i \leq-1 .\end{cases}
$$

Note finally, that $\left\{\Psi_{1}, \Phi_{1}(T)\right\}=\left\{\Psi_{1}, \Phi_{4}(U)\right\}=0$.

Thus we see that $\Psi_{1}$ plays the role of a master-symmetry: taking $\Psi_{2}^{+}, \Psi_{2}^{-}, \Xi_{ \pm 1}^{+}(X), \Xi_{ \pm 1}^{-}(Y)$ for "seeds" and acting by $\left\{\Psi_{1}, \cdot\right\}$, we can obtain the entire hierarchies $\Psi_{i}^{+}, \Psi_{i}^{-}, i>2, \Xi_{i}^{+}(X), \Xi_{i}^{-}(Y)$, $i>1$, and $\Xi_{i}^{+}(X), \Xi_{i}^{-}(Y), i<-1$.

To conclude, let us compare briefly the Lie algebra structures of nonlocal symmetries for all the five linearly degenerate 3D equations studied in [4] and here. All these algebras are infinitedimensional. For the the rdDym equation $u_{t y}=u_{x} u_{x y}-u_{y} u_{x x}$, the 3D Pavlov equation (2), and 
the universal hierarchy equation $u_{y y}=u_{t} u_{x y}-u_{y} u_{t x}$ they are graded. The symmetry algebra of the modified Veronese web equation $u_{t y}=u_{t} u_{x y}-u_{y} u_{t x}$ is filtered (almost-graded). The corresponding algebra for the VWE (1) seemingly admits no reasonable grading or filtering and contains a real irremovable parameter $\lambda$. It will be interesting to study the properties of this algebra in more detail elsewhere.

\section{ACKNOWLEDGMENTS}

Computations were supported by the JETS software, [5].

\section{REFERENCES}

[1] H. Baran, I.S. Krasil'shchik, O.I. Morozov, and P. Vojčák, Symmetry reductions and exact solutions of Lax integrable 3-dimensional systems, J. of Nonlinear Math. Phys., 21, Number 4, 2014, 643-671, arXiv:1407.0246[nlin.SI].

[2] H. Baran, I.S. Krasil'shchik, O.I. Morozov, and P. Vojčák, Integrability properties of some equations obtained by symmetry reductions, J. of Nonlinear Math. Phys., 22, Number 2, 2015, 210-232, arXiv:1412.6461[nlin.SI]

[3] H. Baran, I.S. Krasil'shchik, O.I. Morozov, P. Vojčák, Coverings over Lax integrable equations and their nonlocal symmetries, Theor. and Math. Phys., 188 (2016), 1273-1295, arXiv:1507.00897[nlin.SI].

[4] H. Baran, I.S. Krasil'shchik, O.I. Morozov, and P. Vojčák, Nonlocal Symmetries of Integrable Linearly Degenerate Equations: A Comparative Study, Theor. and Math. Phys., 196 (2018), Issue 2, 1089-1110, arXiv:1611.04938[nlin.SI]

[5] H. Baran, M. Marvan, Jets. A software for differential calculus on jet spaces and diffeties. http://jets.math.slu.cz

[6] A.V. Bocharov et al., Symmetries of Differential Equations in Mathematical Physics and Natural Sciences, edited by A.M. Vinogradov and I.S. Krasil'shchik). Factorial Publ. House, 1997 (in Russian). English translation: Amer. Math. Soc., 1999.

[7] P.A. Burovskiy, E.V. Ferapontov, S.P. Tsarev, Second order quasilinear PDEs and conformal structures in projective space. Int. J. Math. 21 (20106) no. 6, 799-841, arXiv:0802.2626

[8] I.M. Gelfand, I. Zakharevich, Webs, Veronese curves, and bihamiltonian systems, Jouranl of Functional Analysis, 99 (1991) $150-178$

[9] M. Dunajski, A class of Einstein-Weil spaces associated to an integrable system of hydrodynamic type, J. Geom. Phys. 51 (2004) 126-137

[10] M. Dunajski, W. Kryński, Einstein-Weyl geometry, dispersionless Hirota equation and Veronese webs, Math. Proc. Cambridge Philosophical Society 157 (2014) 139-150, arXiv:1301.0621[math.DG]

[11] E.V. Ferapontov, J. Moss, Linearly degenerate partial differential equations and quadratic line complexes, Comm. in Anal. and Geom., 23 (2015) no. 1, 91-127, arXiv:1204.2777 [math.DG]

[12] P. Holba, I.S. Krasil'shchik, O.I. Morozov, P. Vojčák, $2 D$ reductions of the equation $u_{y y}=u_{t x}+u_{y} u_{x x}-u_{x} u_{x y}$ and their nonlocal symmetries, J. of Nonlinear Math. Phys., 24 (2017), Issue 1, 36-47, arXiv:1707.07645[nlin.SI]

[13] P. Holba, I.S. Krasil'shchik, O.I. Morozov, P. Vojčák, Reductions of the universal hierarchy and rdDym equations and their symmetry properties, Lobachevskii J. of Math., 39 (2018) Issue 5, 673-681, arXiv:1712.07063[nlin.SI]

[14] I. Krasil'shchik, Integrability in differential coverings, J. of Geometry and Phys., 87 (2015), 296-304, arXiv:1310.1189[nlin.SI].

[15] I. Krasil'shchik, A. Verbovetsky, R. Vitolo, The Symbolic Computation of Integrability Structures for Partial Differential Equations, Springer Texts \& Monographs in Symbolic Computation, 2017.

[16] I.S. Krasil'shchik and A.M. Vinogradov, Nonlocal trends in the geometry of differential equations: symmetries, conservation laws, and Bäcklund transformations, Acta Appl. Math. 15 (1989) no. 1-2. Also in: A.M. Vinogradov (ed.), Symmetries of partial differential equations. Conservation laws - Applications - Algorithms, Kluwer Acad. Publ., Dordrecht, 1989.

[17] B. Kruglikov, O. Morozov, Integrable dispersionless PDEs in 4D, their symmetry pseudogroups and deformations. Lett. Math. Phys. 105 (2015) 1703-1723, arXiv:1410.7104[math-ph].

[18] B. Kruglikov, A. Panasyuk, Veronese webs and nonlinear PDEs, J. of Geom. and Phys. 115 (2017), 45-60, arXiv:1602.07346 [math.DG]

[19] A. Lelito, O.I. Morozov, Three-component nonlocal conservation laws for Lax-integrable $3 D$ partial differential equations, J. of Geom. and Phys. 131 (2018), 89-100

[20] A.A. Malykh, Y. Nutku, M.B. Sheftel, Partner symmetries and non-invariant solutions of 4-dimensional heavenly equations. J. Phys. A. 37 (2004) 7527-7546, arxiv-math-ph0403020

[21] M. Marvan, Another look on recursion operators, in: Differential Geometry and Applications, Proc. Conf. Brno, 1995 (Masaryk University, Brno, 1996) 393-402.

[22] M. Marvan, A. Sergyeyev, Recursion operators for dispersionless integrable systems in any dimension. Inverse Probl. 28 (2012) no. 2, 025011, arXiv:1107.0784[nlin.SI].

[23] O.I. Morozov, A. Sergyeyev, The four-dimensional Martinez Alonso-Shabat equation: reductions and nonlocal symmetries. J. Geom. Phys. 85 (2014) no. 11, 40-45, arXiv:1401.7942[nlin.SI]

[24] O.I. Morozov, M.V. Pavlov, Bäcklund transformations between four Lax-integrable 3D equations, J. Nonlin. Math. Phys. 24:4 (2017) 465-468 
[25] A. Sergyeyev, A simple construction of recursion operators for multidimensional dispersionless integrable systems. J. Math. Anal. Appl. 454 (2017) no. 2, 468-480, arXiv:1501.01955 [math.AP]

[26] I. Zakharevich, Nonlinear wave equation, nonlinear Riemann problem, and the twistor transform of Veronese webs, arXiv:math-ph/0006001

Trapeznikov Institute of Control Sciences, 65 Profsoyuznaya street, Moscow 117997, Russia \& Independent University of Moscow, B. Vlasevsky 11, 119002 Moscow, Russia

E-mail address: josephkra@gmail.com

Faculty of Applied Mathematics, Agh University of Science and Technology, Al. Mickiewicza 30, Cracow 30-059, Poland \& Trapeznikov Institute of Control Sciences, 65 Profsoyuznaya street, Moscow 117997, Russia

E-mail address: morozov@agh.edu.pl

Mathematical Institute, Silesian University in Opava, Na Rybníčku 1, 74601 Opava, Czech Republic E-mail address: Petr.Vojcak@math.slu.cz 